\title{
ON A PROBLEM OF G. E. SACKS
}

\section{A. H. LACHLAN}

Introduction. On p. 171 of [2] Sacks asks whether there is an r.e. degree of unsolvability $d$ which satisfies $O^{(n)}<d^{(n)}<O^{(n+1)}$ for all $n$. He also conjectures that a proof that such a $d$ exists would use a combinatorial principle not touched on in [2]. In this paper we show using the methods of [2] that such a $\boldsymbol{d}$ exists. ${ }^{1}$ Our proof assumes that the reader has a good understanding of [1] and of the proof of Theorem $3[2$, p. 86].

We present our construction in a semi-formal way in the hope that it will thus be made more comprehensible.

1. Terminology. By function we mean a partial function of natural numbers taking natural number values. We say that the function $\psi$ is an extension of the function $\phi$, written $\phi \subseteq \psi$, if the domain of $\psi$ includes that of $\phi$ and if $\phi$ and $\psi$ agree on the domain of $\phi$. We write $\phi=\psi$ just if $\phi \subseteq \psi$ and $\psi \subseteq \phi ; \phi \neq \psi$ means 'not $\phi=\psi$.' By a finite function we mean one whose doman is finite.

Let $\mathfrak{F}$ denote the class of all singulary partial functions with range $\subseteq\{0,1\}$. By functional we mean a map of $\mathfrak{F}$ into itself; we shall denote functionals by capital Greek letters. The image of a function $\phi$ under a functional $\Psi$ is denoted by $\Psi(\phi)$ and the value of $\Psi(\phi)$ for argument $m$ is denoted by $\Psi(\phi ; m)$. A functional $\Gamma$ is called finite if there is a finite set $Q$ of ordered pairs of finite functions such that $\Gamma(\eta ; x)=y$ if and only if there exists $(\phi, \psi)$ in $Q$ with $\phi \subseteq \eta$ and $\psi(x)=y$. A sequence $\left\{\Gamma_{i}\right\}$ of finite functionals is called strongly recursively enumerable (s.r.e.) if given $i$ we can effectively find a finite set $a_{i}$ of ordered pairs of finite functions which specifies $\Gamma_{i}$ as $Q$ specifies $\Gamma$ above. Similarly, we define an s.r.e. double sequence of finite functionals. A sequence of functionals $\left\{\Psi_{i}\right\}$ is called increasing if for every $\eta$ in $\mathfrak{F}$ and every number $i$ we have $\Psi_{i}(\eta) \subseteq \Psi_{i+1}(\eta)$

We may define a functional to be partial recursive (p.r.) if it can be expressed as the limit (in an obvious sense) of an s.r.e. increasing sequence of finite functionals. Below we shall assume the existence of an s.r.e. double sequence $\left\{\Phi_{i, j}\right\}$ of finite functionals with the following properties. For fixed $i$ the sequence $\Phi_{i, 0}, \Phi_{i, 1}, \cdots$ is increasing, with limit $\Phi_{i}$ say; and $\left\{\Phi_{i}\right\}$ is a standard enumeration of all p.r. functionals.

Received by the editors July 13, 1964.

$1 \mathrm{Mr}$. D. A. Martin has independently obtained an affirmative answer to (Q4) on p. 171 of [2] by an argument different from the one in this paper. 
By the characteristic function of a set of natural numbers we mean the total function which is 0 for arguments in the set and 1 otherwise. We find it convenient to identify sets with their characteristic functions.

Let $S$ be any set, then the sequence of sets

$$
\left\{x \mid \Phi_{i}(S ; x) \text { is defined }\right\}, \quad i=0,1, \cdots,
$$

is a standard enumeration of the sets recursively enumerable in $S$, and the set

$$
\left\{x \mid \Phi_{x}(S ; x) \text { is defined }\right\}
$$

denoted by $S^{\prime}$ is (1-1)-complete for the sets (1) uniformly in $S$. Also, for any set $S$ we let $S^{(0)}=S$ and $S^{(i+1)}=S^{(i) \prime}$ for all $i$. If $S$ has degree $s$, then $S^{\prime}$ has degree $s^{\prime}$.

The empty set is denoted by $\varnothing$, and when we take $S=\varnothing^{(j)}$ the sets (1) are called $j$-enumerable; we adopt the indexing of these sets provided by (1). A set $T$ is called $j$-recursive if both $T$ and its complement $\bar{T}$ are $j$-enumerable; such a set is to be specified by indices of $T$ and $\bar{T}$ as $j$-enumerable sets.

Below we shall require the following elementary result: given a finite functional $\Gamma$, a $j$-recursive set $S$, and numbers $m, n$ such that $\Gamma(S ; m)=n$, we can effectively find assuming a knowledge of $\varnothing^{(j)}$ a number $p$ such that, if $T$ is any set which differs from $S$ only as regards members $\geqq p$, then $\Gamma(T ; m)=n$ also. We say that the number $p$ fixes the value $\Gamma(S ; m)=n$.

For any two sets $S, T$ we let $S \oplus T$ denote the set $\{2 x \mid x \in S\}$ $\cup\{2 x+1 \mid x \in T\}$.

2. Preliminaries. By a close inspection of the proof of Sacks's Theorem 3 [2, p. 86], we obtain:

LEMMA 1. There are recursive functions $\alpha, \beta$ and a p.r. functional $\Theta$ such that, if $e$ is the index of a j-enumerable set $C \oplus \varnothing^{(j)}$ for any $j>0$, then $\alpha(e)$ is the index of a (j-1)-enumerable set $D \oplus \varnothing^{(j-1)}$ such that

$$
\Theta\left(\left(D \oplus \varnothing^{(j-1)}\right)^{\prime}\right)=C \oplus \varnothing^{(j)},
$$

and

$$
\Phi_{\beta(e)}\left(C \oplus \varnothing^{(j)}\right)=\left(D \oplus \varnothing^{(j-1)}\right)^{\prime} .
$$

Of course, Sacks's construction needs a little pulling and pushing to give us this lemma. Roughly speaking, our lemma corresponds to the case of his theorem in which $\boldsymbol{b}=\boldsymbol{g}=O^{(j-1)}$ and in which $\mathbf{c}, \boldsymbol{d}$ are the degrees of $C \oplus \varnothing^{(i)}, D \oplus \varnothing^{(j-1)}$ respectively. The a of Sacks's 
theorem is to be disregarded and the provisions made for it are to be excised. To give more than this meagre explanation would entail repeating a lot of Sacks's proof in a simpler situation.

LEMMA 2. There is a recursive function $\gamma$ with the following property. If $S^{*}, T^{*}$ are sets agreeing with $S, T$ respectively for members $\geqq x$ and if $\Phi_{i}\left(S^{*}\right)=T^{*}$, then $\Phi_{j}(S)=T$ for some $j, j \leqq \gamma(i, x)$.

Proof. There are p.r. functionals $\Psi_{0}^{x}, \Psi_{1}^{x}, \cdots, \Psi_{2^{x}-1}^{x}$ such that, for any set $X, \Psi_{i}^{x}(X)$ with $x$ fixed runs through all sets (meaning all characteristic functions thereof) which agree with $X$ for members $\geqq x$. Hence from $\Phi_{i}\left(S^{*}\right)=T^{*}$ we can infer $\Psi_{m}^{x} \Phi_{i} \Psi_{n}^{x}(S)=T$ for some $m$ and $n$ both $<2^{x}$. But, given $x$, we can effectively construct $\Psi_{0}^{x}, \Psi_{1}^{x}, \cdots, \Psi_{2^{x}-1}^{x}$ and so we can effectively find in terms of $i$ and $x$ a bound for the least index of $\Psi_{m}^{x} \Phi_{i} \Psi_{n}^{x}$.

Lемма 3. There is a recursive function $\gamma$ such that for any sets $S, T$, $\Phi_{i}(S)=T$ implies $\Phi_{\gamma(i)}\left(S^{\prime}\right)=T^{\prime}$.

Proof. By definition we have

$$
T^{\prime}=\left\{x \mid \Phi_{x}(T ; x) \text { is defined }\right\},
$$

and the r.h.s. is just $\left\{x \mid \Phi_{x} \Phi_{i}(S ; x)\right.$ is defined $\}$. We can define a binary recursive function $\alpha$ so that the last set is $\left\{x \mid \Phi_{\alpha(i, x)}(S ; \alpha(i, x))\right.$ is defined $\}$. Thus $\alpha(i, x)$ considered as a function of $x$ constitutes a many-one reduction of $T^{\prime}$ to $S^{\prime}$. We can effectively find the index $\gamma(i)$ of the p.r. functional which maps $\phi$ into the function $\psi$ defined by

$$
\psi(x)=\phi(\alpha(i, x)) .
$$

It is clear that $\Phi_{\gamma(i)}\left(S^{\prime}\right)=T^{\prime}$ as required.

The next lemma combines Lemmas 2 and 3 in a result which we shall use below.

LEMMA 4. There is a ternary recursive function $\gamma$ with the following property. Suppose for $k=0,1$ that $C^{k} \oplus \varnothing^{(j)}$ is the $j$-enumerable set with index $e^{k}$ and that $D^{k} \oplus \varnothing^{(j-1)}$ is the $(j-1)$-enumerable set with index $\alpha\left(e^{k}\right)$. Suppose further that $E^{k}$ agrees with $D^{k} \oplus \varnothing^{(j-1)}$ for members $\geqq x$, and that $\Phi_{i}\left(E^{0}\right)=E^{1}$. Then for some $p, p \leqq \gamma\left(e^{0}, x, i\right)$, we have $\Phi_{p}\left(C^{0} \oplus \varnothing^{(j)}\right)=C^{1} \oplus \varnothing^{(j)}$.

Proof. From Lemma 2 we have

$$
\Phi_{m}\left(D^{0} \oplus \varnothing^{(j-1)}\right)=D^{1} \oplus \varnothing^{(j-1)}
$$

for some $m, m \leqq \gamma(i, x)$. Further, from Lemma 3 


$$
\Phi_{\gamma(m)}\left(\left(D^{0} \oplus \varnothing^{(j-1)}\right)^{\prime}\right)=\left(D^{1} \oplus \varnothing^{(j-1)}\right)^{\prime} .
$$

Using Lemma 1 it follows that

$$
\Theta \Phi_{\gamma(m)} \Phi_{\beta\left(e^{0}\right)}\left(C^{0} \oplus \varnothing^{(j)}\right)=C^{1} \oplus \varnothing^{(j)} .
$$

The required ternary function $\gamma$ can now be defined easily.

We can suppose without loss of generality that $\gamma$ is increasing in all its arguments and also that $\gamma(e, x, i) \geqq i$; these assumptions will be used below.

The singulary function $\alpha$ and the ternary function $\gamma$ both play an important role in the construction to follow.

3. The construction. The idea of the construction is as follows. We construct two sequences $A^{0,0}, A^{0,1}, \cdots ; A^{1,0}, A^{1,1}, \cdots$ of sets so that $A^{0, j}$ and $A^{1, j}$ are $j$-enumerable. Sacks's construction is used to ensure that $A^{k, j+1}$ has the same degree as $\left(A^{k, j}\right)^{\prime}$. Also, we use the method of Friedberg [1] in constructing $A^{0, j}$ and $A^{1, j}$ to ensure that

$$
\Phi_{x}\left(A^{0, y}\right) \neq A^{1, y} \text { and } \Phi_{x}\left(A^{1, y}\right) \neq A^{0, y}
$$

for all $x \leqq j$ and $y \leqq j$. It is clear that, if we can realise these aims, then the problem posed by Sacks is solved.

However, because we have $\Phi_{\beta(e)}$ rather than a fixed p.r. functional in Lemma 1 , we have to proceed in a rather roundabout way. We suppose that we are given (by index) two p.r. functions $\phi^{0}, \phi^{1}$. From these we construct simultaneously two sequences of sets $A^{0,0}$, $A^{0,1}, \cdots$ and $A^{1,0}, A^{1,1}, \cdots$. We shall arrange later for $\phi^{0}, \phi^{1}$ to be recursive and for $A^{k, j+1}$ to be the $(j+1)$-enumerable set with index $\phi^{k}(j)$.

The construction consists of an infinite number of Steps which we number $0,1,2, \cdots$. We denote by $A_{n}^{\mathbf{k}, \boldsymbol{j}}$ the set of numbers enumerated in $A^{k, j}$ in the Steps $\leqq n$. At Step $n$ we shall (among other things) define the auxiliary functions $\delta_{n}^{\boldsymbol{k}, \boldsymbol{j}}, \lambda_{n}, \pi_{n}$ for some arguments; in fact, $\lambda_{n}(x)$ is defined for all $x$ and $\pi_{n}(x)$ is defined for $x \leqq n$.

Step 0 . We set $A_{0}^{0, j}=A_{0}^{1, j}=\varnothing \oplus \varnothing^{(j)}$ and $\lambda_{0}(j)=0$ for all $j$. We set $\pi_{0}(0)=0$.

Step $n+1$. We first compute $\phi^{0}(n)$ and $\phi^{1}(n)$. If either of these is undefined, the construction proceeds no further.

We then carry out the following $2 n+2$ Substeps which are numbered $0,1, \cdots, 2 n+1$. For $n$ even we prescribe:

Substep $2 j$. We seek the least number $e, e \leqq \pi_{n}(j)$, such that either

$$
\Phi_{e, n}\left(A_{n}^{0, j} ; \delta_{n}^{0, j}(e)\right)=A_{n}^{1, j}\left(\delta_{n}^{0, j}(e)\right)=1,
$$

or $\delta_{n}^{1, f}(e)$ is undefined. There are three cases. 
Case 0. If (2) holds, let $p$ be a number, the least say, which fixes the value $\Phi_{e, n}\left(A_{n}^{0, j} ; \delta_{n}^{0, j}(e)\right)$. We define $\lambda_{n+1}(j)$ to be $\operatorname{Max}\left\{p, \lambda_{n}(j)\right\}$; we enumerate $\delta_{n}^{0, j}(e)$ in $A^{1, j}$; also, we define

$$
\begin{aligned}
& \delta_{n+1}^{0, j}(x)=\delta_{n}^{0, j}(x), \\
& \delta_{n+1}^{1, j}(x)= \begin{cases}\delta_{n}^{1, j}(x) & \text { if } x<e, \\
\text { undefined } & \text { if } x \geqq e .\end{cases}
\end{aligned}
$$

Case 1. If (2) does not hold and $\delta_{n}^{1, j}(e)$ is not defined, then we define $\delta_{n+1}^{1, j}(e)$ to be the least even number $\geqq \lambda_{n}(j)$ not in $A_{n}^{0, j}$, and we define $\lambda_{n+1}(j)$ to be $\delta_{n+1}^{1, j}(e)+1$. Also, $\delta_{n+1}^{0, j}$ and $\delta_{n+1}^{i, j}$ are to be the same $\delta_{n}^{0, j}$ and $\delta_{n}^{1, j}$ respectively, except as just provided for $\delta_{n+1}^{n}(e)$.

Case 2. Otherwise, that is, if there is no $e \leqq \pi_{n}(j)$ such that (2) holds or such that $\delta_{n}^{1, j}(e)$ is undefined. Then $\delta_{n+1}^{0, j}, \delta_{n+1}^{1, j}$ are to be the same as $\delta_{n}^{0, j}, \delta_{n}^{1, j}$ respectively.

Substep $2 j+1$. We enumerate in $A^{0, j}$ all the even numbers in the finite set

$$
\left\{x \mid \Phi_{\alpha\left(\phi^{0}(j)\right), n}\left(\varnothing^{(j)} ; x\right) \text { is defined }\right\}
$$

which are $\geqq \lambda_{n+1}(j)$. $\left(\lambda_{n+1}(j)\right.$ is $\lambda_{n}(j)$ if not otherwise defined.)

If $n$ is odd, then Substep $2 j$ is to be obtained from the one given by interchanging the superscripts 0,1 and by interchanging the Cases 0,1 . Thus Case 0 will now read: "If $\delta_{n}^{0, j}(e)$ is not defined, then . . ." Also, in the new Case 1 we must write $x \leqq e, x>e$ for $x<e, x \geqq e$ respectively. In Substep $2 j+1$ we read $A^{1, j}$ for $A^{0, j}$, and $\phi^{1}(j)$ for $\phi^{0}(j)$.

To complete Step $n+1$ we define $\lambda_{n+1}(x)$ to be $\lambda_{n}(x)$ if $\lambda_{n+1}(x)$ has not yet been defined, and we define $\pi_{n+1}(x)$ for $x \leqq n+1$ as follows.

(3)

$$
\begin{aligned}
& \pi_{n+1}(0)=0, \\
& \pi_{n+1}(1)=\gamma\left(\operatorname{Max}\left\{\phi^{0}(0), \phi^{1}(0)\right\}, \lambda_{n+1}(0), 1\right), \\
& \pi_{n+1}(2)=\gamma\left(\operatorname{Max}\left\{\phi^{0}(1), \phi^{1}(1)\right\}, \lambda_{n+1}(1)\right. \text {, } \\
& \left.\gamma\left(\operatorname{Max}\left\{\phi^{0}(0), \phi^{1}(0)\right\}, \lambda_{n+1}(0), 2\right)\right), \\
& \pi_{n+1}(n+1)=\gamma\left(\operatorname{Max}\left\{\phi^{0}(n), \phi^{1}(n)\right\}, \lambda_{n+1}(n),\right. \\
& \gamma\left(\operatorname{Max}\left\{\phi^{0}(n-1), \phi^{1}(n-1)\right\}, \lambda_{n+1}(n-1), \cdots,\right. \\
& \left.\left.\gamma\left(\operatorname{Max}\left\{\phi^{0}(0), \phi^{1}(0)\right\}, \lambda_{n+1}(0), n+1\right) \cdots\right)\right) \text {. }
\end{aligned}
$$

This completes the construction, which can only terminate after a finite number of Steps by $\phi^{0}$ or $\phi^{1}$ being undefined for some argument. If all the Steps $\leqq n$ terminate, then $A_{n}^{0,1}, A_{n}^{1, j}$ are $j$-recursive sets. 
Further, their $j$-recursiveness is uniform in $j$ and $n$ in the sense that given $j$ and $n$ we can effectively find indices of $A_{n}^{0, j}, A_{n}^{1, j}$ and their complements as $j$-enumerable sets. Also, for $k=0,1$ and all $j$ we have $A_{n}^{k, j}$ increasing to the limit $A^{k, j}$ as $n$ increases. This means that $A^{k, j}$ is a $j$-enumerable set. Further, there are recursive functions $\psi^{0}, \psi^{1}$ such that for all $j$ and $k=0,1$ we have $\psi^{k}(j)$ is the index of $A^{k, j+1}$ as a $(j+1)$-enumerable set. Further, indices of $\psi^{0}$ and $\psi^{1}$ can be found effectively given indices of $\phi^{0}$ and $\phi^{1}$. It now follows by the second recursion theorem that we may choose $\phi^{0}, \phi^{1}$ so that $\phi^{0}=\psi^{0}$ and $\phi^{1}=\psi^{1}$. In this case $\phi^{0}, \phi^{1}$ will be recursive, because $\psi^{0}, \psi^{1}$ are. Below we shall suppose that $\phi^{0}$ and $\phi^{1}$ have been so chosen. Thus for $k=0,1$ and all $j, \phi^{k}(j)$ is the index of $A^{k, j+1}$ as a $(j+1)$-enumerable set.

To show that the construction has the hoped-for effect, we first prove:

LEMma 5. For all $j$

(a) $\pi_{n}(j)$ has a limit $\pi(j)$ as $n$ increases;

(b) if $x \leqq \pi(j)$, then $\Phi_{x}\left(A^{0, j}\right) \neq A^{1, j}$ and $\Phi_{x}\left(A^{1, j}\right) \neq A^{0, j}$;

(c) $\lambda_{n}(j)$ has a limit $\lambda(j)$ as $n$ increases.

Proof. We use course-of-values induction on $j$. For $j=0$, (a) is immediate.

Assuming that (a) holds for some $j$ we can prove (b) and (c) for the same $j$. We need only consider the Substeps $2 j, 2 j+1$ at Steps $n+1$ such that $\pi_{x}(j)=\pi(j)$ for all $x \geqq n$. Now Substep $2 j$ is modelled on the construction [1] except that where Friedberg was concerned with all the p.r. functionals we are concerned only with the initial segment $\left\{\Phi_{x} \mid x \leqq \pi(j)\right\}$. In adapting Friedberg's proof we would prove the propositions:

$$
\delta_{n}^{0, j}(x) \text { has a limit } \delta^{0, j}(x) \text { such that }
$$

$$
\delta_{n}^{1, j}(x) \text { has a limit } \delta^{1, j}(x) \text { such that }
$$

$$
\Phi_{x}\left(A^{0, j} ; \delta^{0, j}(x)\right) \neq A^{1, j}\left(\delta^{0, j}(x)\right),
$$

$$
\Phi_{x}\left(A^{1, j} ; \delta^{1, j}(x)\right) \neq A^{0, j}\left(\delta^{1, j}(x)\right)
$$

for $x \leqq \pi(j)$ in the order $(4,0),(5,0),(4,1),(5,1), \cdots,(4, \pi(j))$, $(5, \pi(j))$.

After $\pi_{n}(j)$, and $\delta_{n}^{0, j}(x), \delta_{n}^{1, j}(x)$ for $x \leqq \pi(j)$, have all reached their limits, Case 0 or 1 can only apply in Substep $2 j$ of one further Step. Thus for all but a finite number of Steps Case 2 obtains in Substep $2 j$. Hence $\lambda_{n}(j)$ has a limit $\lambda(j)$. We should have observed already that Substep $2 j+1$ is specifically designed so as not to conflict with the purpose of Substep $2 j$. 
To complete the proof of the lemma we need only remark from the equations (3) that, if $\lambda_{n}(0), \lambda_{n}(1), \cdots, \lambda_{n}(j)$ have the respective limits $\lambda(0), \lambda(1), \cdots, \lambda(j)$, then $\pi_{n}(j+1)$ has the limit

$$
\begin{array}{r}
\gamma\left(\operatorname{Max}\left\{\phi^{0}(j), \phi^{1}(j)\right\}, \lambda(j), \gamma\left(\operatorname{Max}\left\{\phi^{0}(j-1), \phi^{1}(j-1)\right\}, \lambda(j-1), \cdots,\right.\right. \\
\left.\left.\gamma\left(\operatorname{Max}\left\{\phi^{0}(0), \phi^{1}(0)\right\}, \lambda(0), j+1\right) \cdots\right)\right) .
\end{array}
$$

Lemma 6. For all $j$ and $k=0,1, A^{k, j+1}$ is a $(j+1)$-enumerable set of the form $X \oplus \varnothing^{(j+1)}$ with index $\phi^{k}(j)$, and $A^{k, j}$ agrees with the $j$ enumerable set with index $\alpha\left(\phi^{k}(j)\right)$ for members $\geqq \lambda(j)$.

Proof. After Step 0 only even numbers can enter $A^{k, j}$, and so by Step 0 we know that $A^{k, j}$ is of the form $X \oplus \varnothing^{(j)}$. We arranged above for $A^{k, j+1}$ to have index $\phi^{k}(j)$ and so the $j$-enumerable set with index $\alpha\left(\phi^{k}(j)\right)$, call it $W$, has the form $X \oplus \varnothing^{(j)}$ by Lemma 1 . Thus $A^{k, i}$ and $W$ certainly agree for odd members. Now $\lambda(j)$ is the limit of $\lambda_{n}(j)$ and the set

$$
\left\{x \mid \Phi_{\alpha\left(\phi^{k}(j)\right), n}\left(\varnothing^{(j)} ; x\right) \text { is defined }\right\}
$$

increases with $n$ to the limit $W$. Hence by the Substeps $2 j+1$ we have $A^{k, j}$ agreeing with $W$ for members $\geqq \lambda(j)$.

It is clear from Lemmas 1 and 6 that the degree of $A^{k, j+1}$ is the jump of the degree of $A^{k, j}$. To complete the proof it suffices to show that

$$
\Phi_{x}\left(A^{0, j}\right) \neq A^{1, j} \text { and } \Phi_{x}\left(A^{1, j}\right) \neq A^{0, j}
$$

for all $x$ and $j$. When $x \leqq j$, (6) follows by (b) of Lemma 5 , because $\gamma(e, x, i) \geqq i$ which means that $\pi(j) \geqq j$. Now consider the case $x=m$, $j=n$ with $m>n$. Then by Lemmas 4 and 6 we have that

$$
\Phi_{m}\left(A^{0, n}\right) \neq A^{1, n} \text { and } \Phi_{m}\left(A^{1, n}\right) \neq A^{0, n}
$$

holds provided that (6) holds for $j=n+1$ and

$$
x \leqq \gamma\left(\operatorname{Max}\left\{\phi^{0}(n), \phi^{1}(n)\right\}, \lambda(n), m\right) .
$$

However, by another application of Lemma 4 we see that this last condition holds provided that (6) holds for $j=n+2$ and

$$
x \leqq \gamma\left(\operatorname{Max}\left\{\phi^{0}(n+1), \phi^{1}(n+1)\right\}, \lambda(n+1), \gamma\left(\operatorname{Max}\left\{\phi^{0}(n), \phi^{1}(n)\right\}, \lambda(n), m\right)\right) \text {. }
$$

And, repeating the application of Lemma 4 a finite number of times, we can see that (7) will hold provided that (6) holds for $j=m$ and

$$
\begin{array}{r}
x \leqq \gamma\left(\operatorname{Max}\left\{\phi^{0}(m-1), \phi^{1}(m-1)\right\}, \lambda(m-1), \gamma\left(\operatorname{Max}\left\{\phi^{0}(m-2), \phi^{1}(m-2)\right\},\right.\right. \\
\left.\left.\lambda(m-2), \cdots, \gamma\left(\operatorname{Max}\left\{\phi^{0}(n), \phi^{1}(n)\right\}, \lambda(n), m\right) \cdots\right)\right) .
\end{array}
$$


By inspection the r.h.s. of this inequality is $\leqq \pi(m)$ and so $(7)$ follows by (b) of Lemma 5 with $j=m$. This completes the proof of (6).

Taking $a_{0}, a_{1}$ to be the degrees of $A^{0,0}, A^{1,0}$ respectively we have shown that $a_{0}^{(n)}$ and $a_{1}^{(n)}$ are incomparable for all $n$. We may take either $a_{0}$ or $a_{1}$ to be the $d$ whose existence was queried by Sacks.

\section{REFERENCES}

1. R. M. Friedberg, Two recursively enumerable sets of incomparable degrees of unsolvability, Proc. Nat. Acad. Sci. 43 (1957), 236-238.

2. G. E. Sacks, Degrees of unsolvability, Annals of Mathematics Studies No. 55, Princeton Univ. Press, Princeton, N. J., 1963.

University of Newcastle upon Tyne, England 